\title{
Complejidad y empresa familiar
}

\section{Complexity and family business}

\author{
Enrique Claver Cortés ${ }^{\mathrm{a}}$ • Hipólito Molina Manchón ${ }^{\mathrm{a}}$ • Patrocinio Carmen Zaragoza Sáez $^{\mathrm{a}}$ \\ ${ }^{a}$ Departamento de Organización de Empresas. Universidad de Alicante
}

D A T OS A R T Í C U L O

Historial:

Recibido 18-07-2014

Aceptado 26-04-2015

Palabras clave:

Empresa Familiar

Intangibles

Auto-organización

Complejidad

Códigos JEL:

M10

\section{A R T I C L E IN F O \\ Article history: \\ Received 18-07-2014 \\ Accepted 26-04-2015}

Keywords:

Family Firm

Intangibles

Self-organization

Complexity.

JEL codes:

M10

\begin{abstract}
RES UM E N
Tomando como base diversas perspectivas teóricas y la literatura sobre la empresa familiar, este trabajo teórico tiene como objetivo mostrar, mediante un modelo conceptual, el potencial que ofrecen los intangibles que presenta este tipo de organización para hacer frente al fenómeno de la complejidad. De acuerdo con este modelo, concluimos que la empresa familiar reúne las características propias de los sistemas complejos con capacidad de adaptación. Señalamos que este tipo de organización está dotado de un conjunto de intangibles específicos, básicos para el desarrollo de capacidades dinámicas que permitan hacer frente a la complejidad del entorno. La gestión de estas capacidades desde la perspectiva de la complejidad facilita el desarrollo de procesos de auto-organización que dan como resultado innovaciones en diversos ámbitos, permitiendo la adaptación a un entorno complejo.
\end{abstract}

\begin{abstract}
A B S T R A C T
Taking as a reference various theoretical approaches as well as the literature on the family firm, and using a conceptual model, the present theoretical paper has as its aim to show the potential offered by the intangibles present in this type of organization, which allows it to face the phenomenon of complexity. The application of this model leads us to conclude that the family firm gathers the characteristics, which are typical of complex systems with an adaptation capacity. It is highlighted in our research work that such an organisation owns a set of specific intangibles, which turn out to be essential for the development of dynamic capabilities allowing it to cope with the complexity that characterises its business environment. The management of these capabilities from a complexity perspective makes it easier to develop self-organisation processes, which give rise to innovations in a variety of fields, thus permitting the adaptation to a complex environment.
\end{abstract}

\footnotetext{
* Autor de contacto.
}

Correoselectrónicos: enrique.claver@ua.es,hipolito.molina@ua.es*,patrocinio.zaragoza@ua.es 


\section{Introducción}

En los últimos años, en los que el nivel de complejidad del entorno se ha incrementado sustancialmente, ha quedado patente que la ventaja competitiva empresarial se sostiene en activos con un marcado carácter estratégico y en un conjunto de capacidades dinámicas estrechamente vinculadas a la capacidad de aprendizaje de la organización. Podemos decir que las capacidades dinámicas permiten la renovación continua de la base de intangibles (recursos y capacidades) de la empresa, lo cual ayuda enormemente al desarrollo de innovaciones de diversa naturaleza que contribuyen a la supervivencia de la empresa en los mercados.

Por otra parte, de acuerdo con el Instituto de la Empresa Familiar, podemos concebir a las empresas familiares como los principales agentes de creación de riqueza. En España, el número de empresas familiares alcanza la cifra de 2,9 millones (el $85 \%$ sobre el total de empresas), generando 13,9 millones de puestos de empleo, así como el 70\% del PIB. Tienen, por tanto, un considerable impacto económico $\mathrm{y}$, con su actividad, apoyan la creación de cadenas de valor para productos y servicios que representan la mayor parte de la demanda en el mercado. No obstante, en relación al desarrollo de innovaciones, una de las exigencias establecidas por el entorno actual, es de esperar, según el INE (2013), que sean pocas las empresas familiares españolas propensas a innovar. En consecuencia, con el fin de mejorar la gestión de este tipo de empresa, sería interesante conocer qué factores podrían ayudar a impulsar los procesos de innovación como mecanismos que contribuyan a la adaptación a las condiciones de un entorno complejo.

Los argumentos presentados por Lev y Daum (2004) y Kianto (2007) nos conducen a adoptar un enfoque dinámico en torno a los activos intangibles que vienen a representar las capacidades y el potencial necesario para la supervivencia y el crecimiento futuro, enfoque, por otra parte, muy necesario desde la perspectiva de la complejidad. Por esa razón pensamos que dichos activos, constituyentes de lo que se ha venido en llamar capital intelectual de la empresa, podrían jugar un rol importante como motor o dinamizador de los procesos de innovación o de auto-organización, concepto cuyo significado explicaremos más adelante.

Es posible encontrar en la literatura numerosos trabajos tanto teóricos como empíricos sobre el capital intelectual o la empresa familiar, y más teóricos que prácticos acerca de la teoría de la complejidad. Sin embargo, son relativamente pocos los estudios que vinculan el capital intelectual, las capacidades dinámicas y la teoría de la complejidad con las empresas familiares. La falta de investigación en este campo puede deberse a varias circunstancias. En primer lugar, aunque el campo de las capacidades dinámicas comenzó a gestarse hace tiempo, todavía hoy no existe una terminología consistente ni un consenso sobre los factores que favorecen este tipo de capacidades (Wang y Ahmed, 2007). En segundo lugar, la teoría de la complejidad y el estudio de la empresa familiar son dos ámbitos de estudio que han surgido como líneas de investigación con entidad propia hace relativamente poco tiempo. Adicionalmente, en el caso de la teoría de la complejidad, podemos decir, citando a Rodríguez y Aguirre (2011) y a Reynoso (2009), que lo que hoy suele llamarse teoría de la complejidad es en realidad el nombre de un campo con límites borrosos que abarca, en su formulación científica, a las teorías de los sistemas complejos en sentido amplio (sistemas dinámicos, sistemas no lineales, sistemas adaptativos), la teoría del caos y los fractales.

Lo cierto es que, señalan los autores, no existe en la actualidad una teoría unificada de la complejidad que sintetice y sistematice de modo explícito los aspectos fundamentales de las distintas y variadas teorías, métodos y algoritmos de complejidad elaborados en el marco de ciencias y disciplinas diferentes. Por otra parte, en relación a la empresa familiar, el desarrollo de su línea de investigación ha estado limitada por un falta de fuentes de datos secundarios y por la variedad de perspectivas teóricas usadas por los investigadores (Ibrahim, McGuire, Soufani y Poutziouris, 2004).

De acuerdo con lo anterior, este trabajo pretende hacer frente a esa falta de estudios que vinculen el capital intelectual, las capacidades dinámicas y la teoría de la complejidad con las empresas familiares. En este contexto, el objetivo es el de presentar un modelo conceptual (véase la figura 1) que sirva para poner de manifiesto el papel que los intangibles específicos de la empresa familiar pueden desempeñar como factores que contribuyan a su capacidad de adaptación o auto-organización. Concretamente, este objetivo se traduciría en: (1) identificar el vínculo entre capacidades dinámicas y aprendizaje organizativo, dentro del marco establecido por la teoría de la complejidad; y (2) identificar la relación entre los intangibles de la empresa familiar, base de sus capacidades dinámicas, y su capacidad de adaptación, innovación o auto-organización. 
Figura 1. La empresa familiar como sistema complejo con capacidad de adaptación



\section{Marco conceptual: la complejidad ambiental y la complejidad organizativa}

La realidad en la que están inmersas las empresas es actualmente enormemente compleja: existen una amplia multiplicidad de variables que intervienen en la definición de la estructura global de los mercados, variables que, además, se ven alteradas con mucha frecuencia y que interactúan entre sí. Aspectos del entorno vinculados a los rápidos cambios tecnológicos, la desregulación y la globalización de los mercados, la crisis económica y sus efectos, etc. ponen de manifiesto esa complejidad y las dificultades que tienen las empresas, entre otros agentes sociales, para entender lo que está sucediendo.

Comprender realmente todo lo que ocurre es un verdadero reto porque hemos aprendido a ver la realidad que nos rodea con una simplicidad derivada de su fragmentación. Peter Senge (1994) señaló que el hombre no actúa sobre un mundo real, sino que actúa sobre él recurriendo a modelos de la realidad basados en la simplificación. De esta manera, la forma en que se solía hacer frente a la complejidad era fragmentando las cosas en trozos más pequeños, más manejables, en la medida que los directivos de las empresas se veían sujetos al principio de racionalidad limitada y tendían a simplificar la realidad (Simon, 1957). Pero este planteamiento daba por sentado que la suma de las partes era igual al todo, cuando realmente el todo es más que la suma de las partes. Y así es como tradicionalmente intentábamos resolver los problemas del mundo de los negocios, lo que ya no es válido en el mundo en el que nos encontramos.

No obstante, el concepto de complejidad no sólo está vinculado a la situación actual, si no que ya se venía empleando desde que surgió la concepción de la empresa como sistema abierto. Nos estamos refiriendo al concepto de complejidad aplicado tanto a la propia empresa como al entorno que la rodea. La empresa se concibe como una organización compleja (sistema complejo) porque está formada por un conjunto de partes interdependientes que forman un todo relacionado con su entorno (Thompson, 1967). En ella tienen lugar actividades que se dividen, definiendo de esta manera tres tipos de complejidad o diferenciación: horizontal (número de departamentos), vertical (número de niveles jerárquicos) y espacial (número de localizaciones geográficas) (Robbins, 1987). A su vez, el entorno se contempla como una variable determinante de la estructura de la organización o factor de contingencia y, por tanto, de su complejidad interna. 
El entorno que afecta a una empresa puede ser complejo en función del número de elementos que lo configuran, del grado de heterogeneidad de estos elementos y del nivel de interdependencia que mantienen (Duncan, 1972; Child, 1972; Daft, Sormunen, y Parks, 1988). De esta manera, si el número de elementos que integran el entorno es elevado, son todos ellos muy heterogéneos y el nivel de interdependencia es muy alto, el entorno es complejo. En este contexto, el diseño organizativo trata de conseguir el llamado ajuste organizativo o consistencia de los distintos elementos o dimensiones de la organización entre sí (parámetros de diseño), así como la congruencia entre éstos y el contexto existente en la organización (factores de contingencia). Siguiendo a Galbraith (1982), el diseño organizativo tendría como misión encajar la complejidad de la organización con la complejidad del entorno que le afecta, de tal manera que a mayor complejidad en el entorno, mayor complejidad en la organización que le hace frente.

La importancia que ha ido adquiriendo la variable complejidad en el ámbito de la ciencia de la administración de empresas ha hecho que también fuera adquiriendo relevancia para la misma un conjunto de teorías nacidas en el contexto de las ciencias experimentales como la física, la biología o las matemáticas, que se pueden agrupar bajo el término de teoría de la complejidad. Esta teoría se puede considerar, según Rodríguez y Aguirre (2011), como una perspectiva novedosa en la medida en que "introduce, en el terreno de las ciencias, una racionalidad post-clásica que habilita e incorpora problemas ignorados o vedados por el pensamiento científico moderno tales como el desorden, el caos, la no-linealidad, el no-equilibro, la incertidumbre, la contradicción, el azar, la temporalidad, la emergencia, la auto-organización. La teoría de la complejidad puede entenderse, por lo tanto, como un paradigma científico emergente que involucra un nuevo modo de hacer y entender la ciencia, hasta ahora apoyada sobre los principios rectores del mecanicismo, el reduccionismo y el determinismo".

Podemos decir, de acuerdo con lo anterior, que las empresas se pueden concebir como sistemas que presentan un alto grado de complejidad tanto en sus estructuras organizativas como en sus comportamientos y que se desenvuelven en unos entornos que son altamente complejos. La complejidad surge como consecuencia de las continuas interacciones e interrelaciones entre los individuos que integran la organización y entre éstos y los diversos subsistemas de los que forman parte (departamento, empresa) y de los que no forman parte (entorno). En relación con la empresa, por tanto, podemos identificar dos fuentes de complejidad: la complejidad que deriva del entorno y la complejidad que deriva del propio sistema empresa, concretamente, de las distintas personas que lo configuran. Adicionalmente, podemos entender que la complejidad de la empresa surge no sólo vinculada al número de elementos que configuran la empresa y el grado de interrelación e interdependencia entre ellos, si no también relacionada con la necesidad de comprender los problemas a los que se enfrenta, lo que determina la naturaleza y el contenido de las actividades que tienen lugar en su interior. En este contexto conviene recordar la llamada Ley de Ashby de la variedad (se puede entender sinónimo de complejidad) requerida y que se enuncia en los siguientes términos: "sólo la variedad absorbe la variedad" (Ashby, 1956). Viene a manifestar que un sistema complejo debe tener un cierto nivel de variedad o complejidad interna para ser capaz de funcionar y adaptarse dentro de un entorno también complejo; concretamente, no es posible el control de un sistema complejo si no es mediante un sistema regulador tan complejo como el propio sistema dinámico (García y García, 1996). La consecuencia que deriva de esta ley aplicada al ámbito de las organizaciones es la siguiente: una organización debe presentar un nivel de complejidad igual o superior a la complejidad de la realidad a la que se enfrenta para poder adaptarse a la misma. La existencia de un nivel de variedad superior al requerido permitiría disponer de funciones $\mathrm{o}$ recursos que pueden dedicarse a la introducción de innovaciones en el propio entorno (Navarro, 2003).

La empresa se puede concebir con un sistema complejo con capacidad de adaptación. En este sentido, Gell-Mann (1994) define a los sistemas complejos con capacidad de adaptación como aquellos que aprenden y evolucionan de manera semejante a los seres humanos, están constituidos por agentes heterogéneos que se relacionan entre sí y con su entorno de forma no lineal y son capaces de adaptar su comportamiento ilimitadamente apoyándose en su experiencia, comportamiento que no se puede deducir a partir del comportamiento de sus agentes (Holland, 1995).

Siguiendo el planteamiento de Navarro (2003) y Chiva y Camisón (2002), trataremos de exponer las características de los sistemas complejos con capacidad de adaptación.

- Son sistemas alejados del equilibrio como consecuencia de su heterogeneidad y alto grado de 
diferenciación interna y por su continua interacción con el entorno.

- Son sistemas en los que predominan procesos no lineales caracterizados por la desproporcionalidad entre causas y efectos (pequeñas causas no siempre se traducen en efectos pequeños) y por presentar más de una solución posible (causas similares pueden producir diferentes efectos).

- Son sistemas cuya dinámica organizativa resulta imprevisible como consecuencia de los procesos no lineales, de la intervención del azar en el desarrollo organizativo, que a veces presenta tendencias y otras cambios bruscos, y de una combinación de estos dos elementos.

- Son sistemas que se auto-organizan, es decir, reorientan y reforman sus patrones de comportamiento y organización de manera impredecible como consecuencia de la interacción de los agentes que los forman, mediante la mutua adaptación a las necesidades y capacidades cambiantes de los mismos, así como a las demandas y oportunidades cambiantes del entorno, sin que ningún componente en particular dicte el comportamiento colectivo (Stacey, 1996) a pesar de que existan patrones comunes y patrones heterogéneos por parte de cada uno de los agentes. El resultado de la auto-organización es un nuevo orden en la dinámica de respuesta a un entorno complejo y dinámico (Holland, 1995; Kauffman, 1993). Esta heterogeneidad de patrones de conducta permite el aprendizaje y la evolución, en la medida en que facilita cambios en las reglas, fruto de la cooperación $\mathrm{y}$, también, de la competencia entre los agentes y del comportamiento de otros sistemas que forman parte de su entorno.

- Finalmente, ligada a la característica anterior, son sistemas que se caracterizan por su capacidad para adaptarse y para aprender (Holland, 1995). Efectivamente, los sistemas complejos con capacidad de adaptación son creativos cuando se sitúan al "filo", "borde" o "límite del caos" (Stacey, 1996). Se trata de una fase de transición hacia el cambio. El filo del caos se caracteriza porque existe una situación de equilibrio entre la estabilidad y el caos de la que surge una espontánea autoorganización, la creatividad, la adaptación y la innovación (Chiva y Camisón, 2002). Siguiendo las ideas de Prigogine (1997), los sistemas complejos se ven afectados por las fluctuaciones o perturbaciones derivadas de elementos del propio sistema o externos al mismo. En algunas ocasiones, pequeñas variaciones en las fluctuaciones del sistema son despreciables (pequeños efectos), pero cuando las fluctuaciones se amplifican, el sistema se vuelve muy inestable, el sistema está en el límite del caos, punto donde surge la auto-organización que llevará al sistema a otro estado diferente de orden. La intensidad de las fluctuaciones pone a prueba constantemente la estabilidad de un sistema complejo con capacidad de adaptación $\mathrm{y}$, en cierto momento, una o varias de estas fluctuaciones pueden resultar tan fuertes que empujarán al sistema, a través de situaciones de inestabilidad, hacia una estructura totalmente nueva, que, a su vez, será fluctuante y relativamente inestable. La estabilidad de los sistemas complejos con capacidad de adaptación nunca es absoluta, como ya se indicó, puesto que cada sistema está siempre dispuesto a transformarse, a evolucionar. Según Chiva y Camisón (2002) "al filo del caos tiene lugar un proceso de destrucción creativa, en el que el esquema general es reformado o adaptado, debido a las continuas relaciones entre los agentes y su entorno". Podemos entender, de acuerdo con Navarro (2003), que el alejamiento del equilibrio es sinónimo de vitalidad organizativa.

\section{La dirección de la empresa en el contexto de la complejidad y el aprendizaje}

La complejidad exige modificar la manera tradicional de dirigir la empresa ya que los instrumentos convencionales vinculados a las funciones de planificación y gestión no resultan suficientemente operativos. El grado de diversidad en los elementos constitutivos de la complejidad y la interacción entre ellos implican un amplio abanico de situaciones imprevistas, provocan situaciones de alta inestabilidad y dificultan enormemente las posibilidades de predecir y controlar los acontecimientos. Consecuentemente, la complejidad se debe identificar y administrar a través de técnicas o procedimientos que potencien las características intrínsecas de la empresa como sistema complejo con capacidad de adaptación, sobre todo la capacidad de auto-organización.

Por otra parte, la situación actual no permite simplificar la realidad. Si las empresas quieren sobrevivir deben aprehender su complejidad incluso participar en la misma. Para garantizar su continuidad en el mercado, cada empresa debe lograr comprender la complejidad de la realidad que le afecta. No obstante, como ya se señaló anteriormente, se debe tomar en cuenta que la complejidad puede derivar tanto del entorno $\neg-$ complejidad que se aprende y "reduce", según 
Niklas Luhmann (citado por Bustamante y Opazo, 2004)- como desde el propio interior del sistema u organización empresarial -complejidad generada por las personas que la forman-. Pero ¿cómo enfrentarnos a esa complejidad? Gibson, Ivancevich y Donnelly (1999) presentan el aprendizaje organizacional como proceso a través del cual, y como resultado de la experiencia, se produce una comprensión proporcional de las conductas de cambio relativamente duradero. Las empresas pueden recurrir al aprendizaje para tratar de "reducir" la complejidad del entorno que las rodea, pero no debemos malinterpretar esa reducción. La reducción de la complejidad del entorno es un proceso interno de la empresa que supone un proceso de aprendizaje y de cambio estructural que llamamos auto-organización. De acuerdo con este proceso de auto-organización, los límites de la organización se van modificando a medida que surgen cambios en el entorno que la rodea. Esto es resultado del hecho de que las personas que forman la empresa ostentan la capacidad de aprender y de desarrollar capacidades beneficiosas para ella, planteando una complejidad en la que se unen las capacidades destructivas y las constructivas para el sistema (Bustamante y Opazo, 2004). Como consecuencia, la ventaja competitiva debería asentarse cada vez más en activos con un marcado carácter estratégico y en un conjunto de capacidades dinámicas que tienen como denominador común la capacidad de aprendizaje de la organización. La capacidad de aprendizaje, como capacidad dinámica, permite la renovación continua de la base de recursos y capacidades de la empresa, lo cual se materializará en innovaciones tecnológicas (producto y/o proceso) o de mercado (mejora de los servicios a los clientes y/o alcance de nuevos segmentos de mercado), como respuesta a las fluctuaciones o perturbaciones vinculadas a la complejidad que surgen a nivel interno o externo. En este contexto, el aprendizaje organizativo juega un papel fundamental, pues permite desarrollar el conocimiento tácito contenido en las competencias esenciales de la empresa (Nobre, Tobias, y Walker, 2010). De esta manera, apoyándonos en Bueno, Morcillo y Salmador (2006), podemos considerar que las empresas con éxito son aquellas que logran combinar y explotar con habilidad sus competencias y saben proponer respuestas innovadoras adaptadas a las fluctuaciones o perturbaciones vinculadas a la complejidad que surgen a nivel interno o externo. Dicha innovación será el resultado de las capacidades organizativas que permiten nuevos desarrollos tecnológicos, comerciales u organizativos de la empresa, para lo cual será necesario generar nuevo conocimiento (Uotila, Maula, Keil y Zahra, 2009; Jansen, Van den Bosch y Volverda, 2006).

El aprendizaje organizativo ha sido tratado en la literatura desde dos puntos de vista diferentes: la teoría del comportamiento y la teoría cognitiva. Ambos enfoques permiten distinguir dos tipos de aprendizaje.

El aprendizaje operacional, también denominado "aprendizaje incremental" (Miner y Mezias, 1996; Bierly y Chakrabarti, 1996), "aprendizaje en bucle sencillo" (Argyris y Schön, 1978) o "aprendizaje táctico" (Dodgson, 1993), se caracteriza por basarse principalmente en la repetición y por considerar a las empresas como sistemas de adaptación. En él las rutinas organizativas juegan un papel fundamental. El aprendizaje como un proceso adaptativo puede auto-limitar el campo de actuación de la organización, pues no se produce un cambio en el modelo mental o marcos de referencia, impidiendo el descubrimiento de nuevas oportunidades de negocio. La efectividad del aprendizaje en el corto plazo y dentro de contextos familiares puede interferir en el aprendizaje a largo plazo, reduciendo los incentivos para desarrollar competencias con nuevas tecnologías o paradigmas (Levinthal y March, 1993).

El aprendizaje cognitivo, también denominado "aprendizaje radical" (Miner y Mezias, 1996; Bierly y Chakrabarti, 1996), "aprendizaje en doble bucle" (Argyris y Schön, 1978) o "aprendizaje estratégico" (Dodgson, 1993), no sólo conlleva cambios en el comportamiento, sino también cambios cognitivos, es decir, cambio de los modelos mentales. Surge como resultado de un proceso de experimentación cuya interpretación cae fuera del sistema tradicional de valores, rutinas y reglas. Consiste en hacer las cosas de una nueva forma, siendo uno de los requisitos fundamentales "desaprender" las antiguas prácticas, proporcionando experimentación y creatividad.

Ambos tipos de aprendizaje son necesarios en cualquier organización, pues como afirma March (1991), hay que mantener un equilibrio entre la exploración de nuevas prácticas y la explotación de las existentes. Chiva y Camisón (2002) señalan al respecto que el aprendizaje requiere a la vez cambio y estabilidad en su relación entre individuos y entorno ya que demasiado cambio impide ver a los individuos su entorno y demasiada estabilidad dificulta o no induce al aprendizaje. 
Podemos añadir una tercera perspectiva al estudio del aprendizaje organizativo. Ésta es la perspectiva social desde la que se plantea la cultura organizativa como base para la comprensión del aprendizaje organizativo. De acuerdo con este planteamiento, se entiende el aprendizaje organizativo como el proceso que cambia el estado de conocimiento de una organización adoptando una nueva creencia colectiva, modificando una existente, abandonándola $\mathrm{o}$, incluso, alterando el grado de confianza en relación a ésta (Sánchez y Heene, 1997). Por su parte, Cook y Yanow (1996) consideran al aprendizaje organizativo como la adquisición, sustentación o cambio de significados compartidos por las personas que integran la organización a través de artefactos culturales (mitos, símbolos, rituales, etc.) y de las acciones colectivas del grupo.

De acuerdo con lo anterior, podemos señalar que las teorías conductual y cognitivas consideran que el conocimiento es incorporado en la organización a través de las mentes de sus integrantes o considerando que la organización se comporta, en el ámbito del aprendizaje, como lo hacen las personas, mientras que la perspectiva social propone que la introducción del conocimiento se efectúa por medio del trabajo en grupo, los procesos colectivos, o la interacción de sus integrantes (Chiva y Camisón, 2002).

\section{La empresa familiar como sistema complejo y sus capacidades dinámicas}

Para poner de manifiesto el papel que los intangibles específicos de la empresa familiar pueden desempeñar como factores que contribuyan a su capacidad de adaptación o auto-organización, objetivo de este trabajo, es importante clarificar qué entendemos por empresa familiar, vinculando el concepto con la complejidad. Al respecto cabe decir que no existe un criterio unánime generalmente aceptado que sirva para definir a este tipo de empresa, ya que se han utilizado diversos criterios para identificarla. No obstante, el Instituto de la Empresa Familiar (2014) recurre a los siguientes para considerar a una empresa como familiar:

- $\quad$ Propiedad accionarial: La mayoría de las acciones con derecho a voto son propiedad de la persona o personas de la familia que fundó $o$ fundaron la compañía; o son propiedad de la persona que tiene $o$ ha adquirido el capital social de la empresa; o son propiedad de sus esposas, padres, hijo(s) o herederos directos del hijo(s).
- $\quad$ Control: La mayoría de los votos puede ser directa o indirecta.

- Gobierno: Al menos un representante de la familia o pariente participa en la gestión o gobierno de la compañía.

- Derecho de voto: A las compañías cotizadas se les aplica la definición de empresa familiar si la persona que fundó o adquirió la compañía (su capital social), o sus familiares o descendientes poseen el $25 \%$ de los derechos de voto a los que da derecho el capital social.

Además de estos criterios se suele indicar que este tipo de empresas se caracteriza por su vocación de continuidad, es decir, el deseo del fundador $o$ fundadores $\mathrm{y}$ de sus descendientes de que la propiedad, el gobierno y/o la dirección de la empresa permanezcan perpetuamente bajo el control de miembros de la familia.

Por otra parte, cabe decir que la empresa familiar no es ajena a la temática de la complejidad, sino al contrario, tal y como podemos intuir de los criterios que permiten identificarla. Podemos considerar que este tipo de empresa presenta un nivel de complejidad superior al de otros tipos de empresa porque se trata de un sistema que interactúa de una manera muy estrecha con, al menos, una familia, algunos de cuyos miembros desarrollan labores directivas y/o detentan la propiedad de la empresa, miembros que se suceden unos a otros a lo largo del tiempo, de manera que podemos concebir al proceso de sucesión como la fuente principal de complejidad en este tipo de empresa. De acuerdo con esto, podemos señalar que a la complejidad propia de una empresa concebida como sistema abierto, se le añade un grado adicional de complejidad derivado de su estrecha vinculación con una familia. Efectivamente, si seguimos el modelo conceptual de empresa familiar propuesto por Tagiuri y Davis (1996) y denominado "modelo de los tres círculos", podemos contemplar a este tipo de empresa como un sistema complejo con capacidad de adaptación, resultante de la interacción de tres subsistemas: la empresa, la familia y la propiedad, que reúne las características propias de estos sistemas (alejados del equilibrio, que se auto-organizan, con capacidad para adaptarse y aprender, etc.).

Desde la perspectiva de la teoría de los recursos y capacidades, las empresas familiares han sido descritas como inusualmente complejas, dinámicas y ricas en recursos intangibles (Habbershon y Williams, 1999), recursos y capacidades que pueden marcar diferencias sustanciales entre las empresas 
familiares y las que no lo son, traduciéndose tales diferencias en éxito empresarial (Tokarczyk, Hansen, Green y Down, 2007). En este sentido podemos indicar que las ventajas de la empresa familiar se suelen describir como algo específicamente vinculado a una determinada empresa propiedad de una determinada familia. De esta manera, la confluencia del sistema familia y del sistema empresa genera unas capacidades difíciles de imitar o familiness (Chrisman, Chua y Sharma, 2003; Habbershon, Williams y MacMillan, 2003; Habbershon y Williams, 1999). Estas capacidades son difíciles de imitar porque tales interacciones tienen lugar entre los miembros de la familia, la familia como unidad y el negocio, y producen sinergias sistémicas capaces de generar ventajas o desventajas competitivas para la firma (Habbershon, Williams y MacMillan, 2003). En consecuencia, derivadas de elementos como su cultura e historia, las empresas familiares tienen sus propias formas de "hacer las cosas" y unos recursos idiosincrásicos de naturaleza intangible y tácita que conducen a que las empresas familiares, precisamente por su condición familiar, posean activos intangibles que pueden ser la base de sus ventajas competitivas.

Los recursos intangibles y las capacidades se basan en conocimiento y han llegado a constituirse factores muy relevantes en la creación de valor para la empresa (Lev y Daum, 2004) debido a su valor estratégico. La visión de la empresa basada en el capital intelectual (intellectual capital-based view) se centra exclusivamente en el análisis de aquellos recursos y capacidades de carácter intangible, prestando especial atención a los stocks y flujos de conocimiento incorporados en la empresa (Reed, Lubatkin y Srinivasan, 2006). Por otra parte, el capital intelectual puede definirse como la suma del conocimiento y de las capacidades de conocimiento que la empresa puede usar para obtener ventajas competitivas (Youndt, Subramaniam y Snell, 2004).

Al igual que Lev y Daum (2004), pensamos que los intangibles representan capacidades y potencial para el crecimiento futuro. Consideramos que estos intangibles intervienen en el desarrollo de capacidades (Sirmon y Hitt, 2003) y, por sus características, pueden facilitar tanto el aprendizaje como la innovación. No obstante, conviene señalar que la gestión que se haga de algunos de esos recursos específicos (vinculados con el estilo de liderazgo adoptado por la familia a lo largo del tiempo) puede afectar negativamente tanto al proceso de aprendizaje como a la capacidad innovadora de la empresa.
Siguiendo la línea de razonamiento que venimos manteniendo, a continuación, ofrecemos una descripción de los principales intangibles de la empresa familiar, a la vez que planteamos, desde la perspectiva de la complejidad, una serie de consideraciones vinculadas a su gestión de manera que contribuyan a potenciar su capacidad de adaptación y de aprendizaje, así como el desarrollo de procesos de auto-organización.

Aunque resulta muy complejo determinar la existencia de un modelo particular que diferencie la actividad innovadora de las empresas familiares de las que no lo son, siguiendo a Quintana (2005) podemos indicar que hay un factor presente únicamente en las empresas familiares con una gran incidencia en su capacidad innovadora: la figura del fundador/emprendedor. Este personaje es el que determina la disposición a invertir en nuevos conocimientos y a encontrarles aplicaciones prácticas.

Las motivaciones y las características personales del fundador/emprendedor, como, por ejemplo, su predisposición al cambio, a aceptar nuevas ideas y llevarlas a la práctica, a interactuar con los demás miembros de la organización, etc., favorecerán los procesos de auto-organización y le darán la capacidad para encontrar problemas y resolverlos, aprovechar las oportunidades que ofrecen los mercados, fijar objetivos, controlar su destino, buscar prestigio y reconocimiento, aunque no siempre su fin último sea la obtención de beneficio. Quintana (2005) identifica tres tipos de emprendedores innovadores: el científico o tecnólogo, que detecta una oportunidad de negocio e impulsa la innovación aprovechando sus propios conocimientos o generando los conocimientos necesarios; el emprendedor que detecta una oportunidad y busca en el exterior los conocimientos que permitan su aprovechamiento a través de la contratación del personal adecuado o buscando socios que compartan la búsqueda de esos conocimientos, reservándose la gestión del negocio, y el emprendedor que crea el entorno de trabajo, las condiciones laborales idóneas donde florecen los procesos creativos. Para este autor el tercer patrón es sostenible a lo largo del tiempo, debiendo confluir los dos primeros en este último, dado que es muy complejo tanto sostener la capacidad innovadora permanentemente sobre los conocimientos de la familia, como basarla fundamentalmente en conocimientos externos. En definitiva, se necesita un líder que contribuya al cambio empresarial, generando una capacidad auto-organizadora en la empresa y un líder que agrupe a su alrededor un 
equipo formado por personas capaces de dentro y fuera de la familia, que los combine y los coordine de forma adecuada, que contribuya a la interacción interpersonal, que les proporcione capacidad decisoria en función de sus aptitudes y conocimientos y que se aleje de comportamientos paternalistas, ya que estos últimos, al interferir en las decisiones y la autonomía de esas personas capaces, provoca con frecuencia resistencia al cambio, reduciendo las capacidades dinámicas y la creación de valor (Chirico, Nordqvist, Colombo y Mollona, 2012).

Muy vinculado a la figura del fundador se encuentra el espíritu emprendedor. Sin un espíritu emprendedor bien definido y asentado, no habría empresas familiares, ya que, como señalan Bañegil, Barroso y Tato (2011), "el espíritu emprendedor es un concepto útil que conduce a las empresas en cómo participan en el cambio y en la renovación de procesos para mantener y mejorar su competitividad y continuidad". Uno de los principales problemas que suele afectar a la empresa familiar y que incide sobre su capacidad innovadora, es el acomodamiento en una situación exitosa determinada y la pérdida del espíritu emprendedor, lo que supone una predominancia del denominado aprendizaje operacional o adaptativo. Efectivamente, el éxito, vinculado a una situación concreta, hace que la empresa familiar tienda a especializarse en esa situación, a que su visión estratégica se reduzca considerablemente, a que la cultura vigente en ese momento se refuerce y a que caiga en la autocomplacencia. En definitiva, el éxito de este tipo de empresa hace que se vuelva menos compleja con lo que pierde capacidad de aprendizaje y de adaptación. Para hacer frente a este problema, Gallo y Amat (2003) proponen la adopción de un planteamiento estratégico formal que incorpore este espíritu y la adopción de mecanismos de gobierno familiar que contribuyan a su transmisión a futuras generaciones. Entre esos mecanismos resalta la planificación de la sucesión y el proceso de profesionalización de la empresa. Con ellos la familia propietaria contará con familiares capacitados para la dirección en un contexto de complejidad, puesto que habrán asumido valores vinculados al espíritu emprendedor. También incorporará ejecutivos profesionales ajenos a la familia que contribuyan a renovar el estilo de liderazgo y orientarlo hacia aquel que más facilite el desarrollo de los procesos de auto-organización. De esta manera tendrá lugar el aprendizaje radical, cambiando la forma de hacer las cosas en la empresa y desaprendiendo las antiguas prácticas.
En la mayoría de las ocasiones, y estrechamente vinculados a los procesos de sucesión y profesionalización, se encuentra la implantación del Protocolo familiar en virtud del cual, según el tamaño del grupo y la dimensión de la familia, se establecen órganos de gobierno tales como la Asamblea familiar, el Consejo de la Holding familiar y los Consejos familiares de las empresas del grupo. De acuerdo con Nueno (2011), "el buen diseño de estas estructuras de gobierno y la regulación acordada por la familia para disponerlos y renovarlos, son el motor de una evolución empresarial que requiere una renovación continua. Esta renovación implica innovar buscando la oportunidad, invertir para transformarla en negocio y desinvertir cuando la oportunidad languidece. Con el gobierno adecuado, este proceso emprendedor puede mantenerse a través de generaciones".

La cultura de la empresa familiar suele estar muy determinada tanto por lo que el fundador/emprendedor haya establecido en sus inicios como por la cultura de la propia familia. Los valores que expresan los miembros de la familia, su nivel de compromiso, sentimiento de pertenencia y dedicación, a los que también podemos considerar intangibles de gran valor, crean un propósito común en los empleados y ayudan a establecer en ellos un sentimiento de identificación y compromiso.

La identidad compartida entre los miembros de la empresa, vinculada a la cultura existente, permite aumentar la lealtad familiar y empresarial, gracias a lo cual se obtiene un fuerte sentimiento de misión y se adoptan decisiones empresariales más objetivas. Además, los individuos se sienten propicios a cooperar, comunicar y compartir información y conocimientos (Cibrián, Hernández y Guallarte, 2012), generándose un clima favorable hacia los procesos de auto-organización. Efectivamente, tal y como señala la perspectiva social del aprendizaje organizativo, a la cultura de la empresa se le considera su base explicativa. Los significados que establece la cultura de una empresa y que se concretan en actos, lenguaje u objetos (artefactos culturales) son el medio a través del cual el conocimiento colectivo se transmite y expresa. Para Chiva y Camisón (2002) "las organizaciones están continuamente modificando y manteniendo dichos significados, es decir, cambiando y preservando su identidad cultural, lo cual implica aprendizaje organizativo". De acuerdo con esto, dado que, en general, la cultura de la empresa familiar está bien definida y es conocida por todos, la misma puede contribuir al desarrollo de procesos de cambio, sobre todo si lleva una impronta emprendedora derivada 
del carácter del fundador o contiene algunos aspectos relevantes con incidencia positiva en la capacidad innovadora tales como su orientación a largo plazo (Harris, Martinez y Ward, 1994), la preocupación por la calidad (el nombre de la familia está comprometido con los productos de la empresa), es fuente de orgullo y estabilidad (Kets de Vries, 1993; Davis, 1983), la creencia de que los empleados satisfechos son siempre buenos empleados (Donckels y Fröhlich, 1991).

En definitiva, los valores o significados que integran la cultura de la empresa familiar, influyen en las conductas y actitudes de sus miembros (Rodríguez y Rodríguez, 2013) ya que sirven como elemento orientador en la toma de decisiones o en la operativa a desarrollar por el individuo sin necesidad de que éste se vea obligado a seguir un procedimiento determinado o a alcanzar unos resultados especificados. De acuerdo con esto podemos decir que, en general, las empresas familiares, desde el punto de vista de la cultura, pueden presentar aptitud para el desarrollo de procesos de auto-organización en la medida en que pueden ofrecer condiciones para la experimentación, la improvisación, la creatividad, los pequeños cambios, la búsqueda de soluciones y la tolerancia (Chiva y Camisón, 2003).

La historia de la empresa familiar, muy vinculada a su cultura, recoge su memoria relativa a las decisiones pasadas. Es decir, indica cómo se actuó en el pasado ante determinadas situaciones o con qué estrategias se hizo frente a amenazas o se aprovecharon oportunidades. La gestión que se haga de este recurso puede perjudicar o favorecer la capacidad para la auto-organización de la empresa familiar. Resulta perjudicial si, de acuerdo con esta historia, la empresa familiar opta por actuar como lo viene haciendo tradicionalmente y mantener los mismos productos o servicios, o con modificaciones poco relevantes (aprendizaje adaptativo). Por el contrario, esta experiencia pasada se traduce en un conocimiento específico de los miembros de la familia que favorece una actuación proactiva, rápida y flexible de la empresa si, en virtud de la misma, éstos han asumido que siempre se ha actuado, en todos los aspectos de la empresa, buscando, generando y aprovechando nuevos conocimientos. Estos nuevos conocimientos sirven como fuente de inspiración para nuevos productos y servicios, aunque eso suponga suprimir los productos o los servicios que la situaron en el pasado en una posición estratégicamente ventajosa y con los que puede existir, por tanto, un vínculo emocional.
Para Sirmon y Hitt (2003) la creatividad, las habilidades, las capacidades y el conocimiento adquiridos de los miembros de la familia permite que las empresas familiares se caractericen por la existencia de un gran potencial para generar un profundo conocimiento tácito específico de la empresa y difícil de imitar.

Muchas empresas familiares han sido creadas en la habitación de costura de la casa familiar, en la cocina, en el garaje, en el patio, en un pequeño taller, en una tienda de ropa de barrio, etc. Lugares en los que el padre, la madre, el abuelo o la abuela desarrollaban pequeños trabajos domésticos o profesionales fundamentados, en muchas ocasiones, en unos conocimientos adquiridos de los antepasados. Estas pequeñas actividades, con o sin repercusión económica en sus inicios, terminaron convirtiéndose en el germen de un nuevo negocio, el cual ha sobrevivido, crecido y diversificado. Sólo hay que revisar la historia de muchas empresas familiares que encontramos hoy en día en el mercado.

De acuerdo con lo anterior, podemos afirmar que en el origen de la empresa familiar se encuentra un conocimiento o competencias nucleares (un saber hacer privativo, exclusivo de la familia) que se han transmitido de padres a hijos de manera tácita y que se han ido enriqueciendo con nuevos conocimientos aportados por unos descendientes y empleados bien formados y bien relacionados con determinados agentes del entorno. Es decir, ese conocimiento inicial se ha ido renovando continuamente para hacer frente a las nuevas exigencias impuestas por el entorno empresarial. Las nuevas ideas, los nuevos conocimientos que ofrecen oportunidades, producen perturbaciones en la organización que inducen al desarrollo de procesos de auto-organización, lo que provoca la sustitución de un viejo modelo de comportamiento por uno nuevo.

Con carácter general, la estructura organizativa de las empresas familiares resalta por su carácter orgánico (menos burocrático e impersonal) ya que, la mayoría de estas empresas son de reducida dimensión. Este tamaño provoca, en general, un menor grado de especialización horizontal en sus miembros. Nos encontramos, por tanto, con integrantes polivalentes puesto que desempeñan una amplia variedad de tareas, con cualificaciones múltiples y que, por ello, son muy valiosos para la empresa (Navarro, 2003). Por otra parte, son estructuras en las que las decisiones, dependiendo del tamaño de la organización, suelen estar centralizadas en los miembros de la familia que 
ocupan los niveles más altos de la jerarquía (Goffee y Scase, 1985; Hall, 1988; Tagiuri y Davis, 1996; Allio, 2004), con pocos niveles jerárquicos, de fácil acceso desde los niveles inferiores y con una comunicación formal e informal fluida que permite rapidez y eficacia en la toma de decisiones y en su puesta en práctica. Por todo lo anterior podemos señalar que son estructuras con un alto grado de flexibilidad (Kets de Vries, 1993) o capacidad de adaptación.

Tal y como señalan Björnberg y Nicholson (2012), la supervivencia de las empresas familiares depende de la participación y la inclusión de la próxima generación de miembros de la familia. Ya sea como empleados o propietarios, el compromiso y la voluntad de la próxima generación es la clave para la continuidad de la empresa familiar. Por ello, en una empresa familiar se deberá prestar especial atención a las relaciones entre padres e hijos, por su influencia sobre las actitudes y el comportamiento de éstos (Mussolino y Calabrò, 2014) cuando se incorporan a la empresa familiar con responsabilidades directivas, o entre sucesores miembros de la familia, cuando comparten la dirección de la firma. No obstante, estos cambios en el ámbito generacional pueden ser fuente tanto de ventajas como de inconvenientes en materia de capacidad de adaptación.

El origen de conflictos intergeneracionales puede derivarse de que el fundador no está dispuesto a compartir el poder, es generalmente autoritario y estratégicamente conservador, mientras que los posibles sucesores son más propensos al cambio estratégico, desean una independencia personal y una oportunidad para probar su valor (Sharma et al., 1997). De igual manera, las relaciones entre sucesores pueden ser muy intensas y, si se plantean en términos de discordia permanente, el efecto sobre la empresa puede ser nefasto, ya que se altera el proceso de dirección y el buen clima de la empresa.

No obstante, el cambio de situación que se plantea en estos dos casos puede contribuir enormemente a la capacidad de auto-organización ya que aporta diversidad de opiniones y perspectivas de análisis de los problemas, trabajo en equipo y mayor objetividad en la evaluación y la toma de decisiones (Quintana, 2005), siempre que el fundador, para el primer caso, esté dispuesto a compartir la dirección de la empresa y lo que ello supone o, en el segundo caso, si los sucesores han sido capaces de adquirir las habilidades interpersonales necesarias para la toma de decisiones compartida. Es evidente que con todo ello se conserva la fuerza de la empresa familiar y se mantiene la energía que alimenta el entusiasmo por su crecimiento (Ward, 1999).

Diversos autores han argumentado la existencia en la empresa familiar de un conjunto de valores vinculados con la responsabilidad social (Miller y Le Breton-Miller, 2003; Neubauer y Lank, 1998; Ward, 1987) dado que este tipo de empresa se suele considerar como un miembro más de la sociedad. De acuerdo con ello, la empresa familiar no es una organización aislada, sino una organización compleja dentro de la sociedad que debe actuar de modo solidario lo que significa contribuir de modo inteligente y eficaz al bien común. De igual manera, la empresa familiar tiene un fuerte componente social que afecta a las decisiones que determinan su estrategia, sus operaciones y su estructura. Las empresas familiares, al igual que las no familiares, deben prestar especial atención a las relaciones que mantienen con los agentes de su entorno y que vienen constituidos por los grupos de interés o stakeholders.

La responsabilidad social asumida, el componente social y los grupos de interés se configuran como el origen de fluctuaciones o perturbaciones vinculadas a la complejidad interna y/o externa que conducen a procesos de autoorganización que se resolverán con un nuevo tipo de innovación: la innovación para el desarrollo sostenible.

Esta innovación estará relacionada con el desarrollo de nuevos negocios, productos y servicios que contribuyan una sociedad mucho más sostenible. Al respecto señala el Instituto de Empresa Familiar (2011) que "la empresa familiar cuenta con las herramientas necesarias para situarse favorablemente en este nuevo contexto y poder liderar la transición hacia la sostenibilidad: los valores vinculados a la creación de proyecto a largo plazo, la estructura intergeneracional que les otorga una visión también a largo plazo, así como a la construcción, el fortalecimiento y el mantenimiento del sentido de grupo y la pertenencia a una colectividad con fines que van mucho más allá del interés económico exclusivo a corto plazo".

\section{Conclusiones}

La revisión de este trabajo nos permite plantear unas conclusiones con implicaciones para el ámbito de la empresa familiar. En primer lugar, desde la perspectiva de la teoría de la complejidad, la empresa familiar es un tipo de organización que presenta un nivel de complejidad superior a aquella 
empresa que no tiene la consideración de familiar. Ello hace que pueda reunir las características propias de los sistemas complejos con capacidad de adaptación, es decir, sistemas alejados del equilibrio en los que predominan procesos no lineales, lo que provoca una dinámica organizativa imprevisible, y que se auto-organizan, lo que les dota de capacidad de aprender y de adaptarse. En segundo lugar, desde el planteamiento de la teoría de los recursos y capacidades, las aportaciones de la perspectiva basada en el capital intelectual y la literatura de la empresa familiar, este tipo de organización, con carácter general, está dotada de un conjunto de intangibles específicos que pueden servir de base para el desarrollo de capacidades dinámicas y que, en este sentido, ofrece un considerable potencial para hacer frente a la complejidad del entorno. De acuerdo con estas dos conclusiones, los responsables de las empresas familiares podrían ocuparse de la gestión de esas capacidades específicas desde la perspectiva de la complejidad, con el objetivo de potenciar el desarrollo de procesos de autoorganización. Ello permitirá crear las condiciones necesarias que faciliten la generación de innovaciones en diversos ámbitos de la organización, lo que, a su vez facilitará la adaptación a un entorno complejo.

En cuanto a las contribuciones, con este trabajo creemos que, desde una perspectiva teórica, hemos conseguido mostrar en cierta medida, mediante un modelo conceptual, el potencial que ofrecen los intangibles que presenta la empresa familiar para hacer frente al fenómeno de la complejidad. También pensamos que, desde un punto de vista directivo, nuestra propuesta de gestionar los intangibles de este tipo de empresa, desde la perspectiva de aprehender la complejidad en beneficio propio, teniendo siempre en cuenta de qué manera los intangibles pueden favorecer los procesos de auto-organización, puede dotarla de una considerable capacidad de aprendizaje y adaptación.

Por otra parte, somos conscientes de que este trabajo presenta limitaciones derivadas principalmente de su naturaleza teórica y de su falta de contrastación con evidencia empírica. No obstante, estas limitaciones nos motivan a seguir trabajando en esta línea de investigación que creemos novedosa y que ofrece importantes implicaciones para la gestión de las empresas familiares en el ámbito de la complejidad, con el fin de desarrollarla, ampliarla y pasar a un segundo nivel, en el que no solamente se ofrezca un modelo conceptual quizá más consistente, sino una contrastación empírica que verifique realmente el papel de la gestión "compleja" de los intangibles en el desarrollo de procesos de auto-organización.

\section{Bibliografía}

Allio, M.K. (2004). Family business: their virtues, vices, and strategic path. Strategy \& Leadership, 32(4), 24-33.

Argyris, C. y Schön, D. (1978). Organizational learning. MA: Addison-Wesley.

Ashby, W.R. (1956). An introduction to cybernetics. Londres: Chapman and Hall.

Bañegil Palacios T.M., Barroso Martínez, A. y Tato Jiménez, J.L. (2011): Profesionalizarse, emprender y aliarse para que la empresa familiar continue. Revista de Empresa Familiar, 1(2), 27-41.

Bierly, P. y Chakrabarti, A. (1996). Generic knowledge strategies in the USA. Pharmaceutical Industry. Strategic Management Journal, 17(winter special issue), 123-135.

Björnberg, Å. y Nicholson, N. (2014): Emotional ownership: The next generation's relationship with the family firm. Family Business Review, 25(4), 374-390.

Bueno, E., Morcillo, P. y Salmador, M.P. (2006). Dirección estratégica. Nuevas perspectivas teóricas. Madrid: Pirámide.

Bustamante, M.A. y Opazo, P.A. (2004). Hacia un concepto de complejidad: sistema, organización y empresa. Serie Documentos Docentes FACE SDD, No. 03, Año 2, diciembre, Chile: Universidad de Talca. Extraido el 17 de marzo de 2014, de http://www.panorama.utalca.cl/dentro/sdd/hacia un conc epto.pdf

Child, J. (1972). Organization, structure, environment and performance: The role of strategic choice. Sociology, 6(1), 1-22.

Chirico, F., Nordqvist, M., Colombo, G. y Mollona, E. (2012). Simulating dynamic capabilities and value creation in family firms: Is paternalism an "asset" or a "liability"? Family Business Review, 25(3), 318-338.

Chiva Gémez, R. y Camisón Zornoza, C. (2002). Aprendizaje organizativo y teoría de la complejidad: implicaciones en la gestión del diseño del producto. Castellón de la Plana: Publicacions de la Universitat Jaume I.

Chrisman, J.J., Chua, J.H. y Sharma, P. (2003). Current trends and future directions in family business management studies: toward a theory of the family firm. Coleman White Paper Series. Coleman Foundation and U.S. Association of Small Business and Entrepreneurship.

Cibrián Llanderal, T., Hernández Calzada, M. A. y Guallarte i Nuez, C. (2012). Recursos de capital social y capacidades estratégicas en la empresa familiar. Ponencia 
presentada al XXVI Congreso Anual AEDEM, Barcelona.

Cook, S. y Yanow, D. (1996). Culture and organizational learning. En M. Cohen y L. Sproull (Eds.), Organizational learning (pp. 430-459). California: Sage Publications.

Daft, R.L., Sormunen, J. y Parks, A. (1988). Chief executive scanning, environmental characteristics and company performance: An empirical study. Strategic Management Journal, 9(2), 123-139.

Davis, P. (1983). Realizing the potential of the family business. Organizational Dynamics, 12(1), 47-56.

Dodgson, M. (1993). Organizational learning: A review of some literatures. Organization Studies, 14(3), 375-394.

Donckels, R. y Fröhlich, E. (1991). Are family businesses really different? European experiences from STRATOS. Family Business Review, 4(2), 149-160.

Duncan, R. (1972). Characteristics of organizational environment and perceived environment uncertainty. Adminstrative Sciencie Quaterly, 1(17), 313-327.

Galbraith, J.R. (1982). Designing complex organizations. Reading: Addisson Wesley.

Gallo, M.A. y Amat, J.M. (2003). Los secretos de las empresas familiares centenarias. Claves del éxito de las empresas familiares multigeneracionales. Barcelona: Deusto.

García Carrasco J. y García del Dujo, A. (1996). Teoría de la educación. Salamanca: Ediciones Universidad de Salamanca.

Gell-Mann, M. (1994). El quark y el jaguar. Aventuras en lo simple y en lo complejo. Barcelona: Metatemas.

Gibson, J.L., Ivancevich, J.M. y Donnelly, J.H. (1999). Las organizaciones: Comportamiento, estructura, procesos. Irwin: MacGraw Hill.

Goffee, R. y Scase, R. (1985). Propietorial control in family firms: Some functions of cuasi-organic management systems. Journal of Management Studies, 22(1), 53-68.

Habbershon, T.G. y Williams, M.L. (1999). A resource-based framework for assessing the strategic adventages of family firms. Family Business Review, 12(1), 1-25.

Habbershon, T.G., Williams, M. y MacMillan, I.C. (2003). A unified systems perspective of family firm performance. Journal of Business Venturing, 18(4), 451465.

Hall, P.D. (1988). A historical overview of family firms in the United States. Family Business Review, 1(2), 51-68.

Harris, D., Martinez, J.I. y Ward, J.L. (1994). Is strategy different for the family-owned business? Family Business Review, 7(2), 159-174.
Holland, J.H. (1995). Hidden order. How adaptation builds complexity. Reading: Addison-Wesley.

Ibrahim, A.B., McGuire, J., Soufani, K. y Poutziouris, P. (2004), Patterns in strategy formation in a family firm. International Journal of Entrepreneurial Behaviour \& Research, 10(1/2), 127-140.

INE (2013): Encuesta sobre innovación en las empresas 2012. Extraido el 15 de enero de 2014, de http://www.ine.es/jaxi/menu.do?type $=$ pcaxis \&path $=\% 2 \mathrm{Ft}$ $14 \% 2 \mathrm{Fp} 061 \&$ file $=$ inebase $\& \mathrm{~L}=0$

Instituto de la Empresa Familiar (2011). Innovando para el desarrollo sostenible. Mejorando la competitividad y la productividad de las empresas familiares. Documento 162. Extraido el 9 enero de 2014, de (http://www.iefamiliar.com/web/en/estudios/2011/estudio .html

Instituto de la Empresa Familiar http://www.iefamiliar.com/web/es/ief.html

Jansen, J.P., Van den Bosch, F.A. y Volverda, H.W. (2006). Exploratory innovation, exploitative innovation and performance: Effects of organizational antecedents and environmental moderators. Management Science, 52(11), 1661-1674.

Kauffman, S.A. (1993). The origins of order: Selforganization and selection in evolution. Nueva York: Oxford University Press.

Kets de Vries, M.F.R. (1993). The dynamics of family cotrolled firms: The good and the bad news. Organizational Dynamics, 21(3), 59-71.

Kianto, A. (2007). What do we really mean by the dynamic dimension of intellectual capital? International Journal of Learning and Intellectual Capital, 4(4), 342356.

Lev, B. y Daum, J. (2004). The dominance of intangible assets: Consequences for enterprise management and corporate reporting. Measuring Business Excellence, 8(1), 6-17.

Levinthal, D. y March, J. (1993). The myopia of learning. Strategic Management Journal, 14 (winter special issue), 95-112.

March, J. (1991). Exploration and exploitation in organizational learning. Organization Science, 2(1), 7187.

Miller, D. y Le Breton-Miller, I. (2003). Challenge versus advantage in family business. Strategic Organization, 1(1), 127-134.

Miner, A. y Mezias S. (1996). Ugly duckling no more: Pasts and futures of organizational learning research. Organization Science, 7(1), 88-99.

Mussolino, D. y Calabrò, A. (2014). Paternalistic leadership in family firms: Types and implications for intergenerational succession. Journal of Family Business Strategy, 5(2), 197-210. 
Navarro Cid, J. (2003). Gestión de organizaciones: gestión del caos. Dirección y Organización, 23, 136-145.

Neubauer, F. y Lank, A.G. (1998). The family business: its governance for sustainability. London: McMillan Press.

Nobre, F.S., Tobias, A.M. y Walker, D.S. (2010). A new contingency view of the organization: Managing complexity and uncertainty through cognition. Brazilian Administration Review, 7(4), 379-396.

Nueno, P. (2011). Iniciativa emprendedora y empresa familiar: Emprendiendo a través de las generaciones. Universia Business Review, cuarto trimestre, 96-101.

Prigogine, I. (1997). Las leyes del caos. Barcelona: Drakontos.

Quintana, J. (2005). La innovación en las empresas familiares. CLM. Economía, Vol. 7, 103-130. Extraido el 22 de diciembre de 2013, de www.clmeconomia.jccm.es/pdfclm/quintana_clm7.pdf

Reed, K.K., Lubatkin , M. y Srinivasan, N. (2006). Proposing and testing an intellectual capital-based view of the firm. Journal of Management Studies, 43(4), 867-893.

Reynoso, C. (2009). Modelos o metáforas. Crítica del paradigma de la complejidad de Edgar Morin. Buenos Aires: Editorial SB.

Robbins, S.P. (1987). Organization theory: Structure, design and applications. Nueva York: Prentice Hall.

Rodríguez Zapatero, M. y Rodríguez Jiménez, M. (2013). Los valores en la empresa familiar: análisis de su influencia en el comportamiento. Revista de Empresa Familiar, 3(2), 7-16.

Rodríguez Zoya, L. G. y Aguirre, J.L. (2011). Teorías de la complejidad y ciencias sociales. Nuevas estrategias epistemológicas y metodológicas. Nómadas. Revista Crítica de Ciencias Sociales y Jurídicas, 30(2). Extraido el 11 de diciembre de 2013, de http://www.ucm.es/info/nomadas/30/rdzzoya_aguirre.pdf

Sanchez, R. y Heene, A. (1996). A systems view of the firm in competence based competition. En R. Sanchez, A. Heene y H. Thomas (Eds.), Dynamics of competence-based competition: Theory and practice in the new strategic management (39-62). Oxford: Elsevier Pergamon.
Senge, P. (1994). The firth discipline fieldbook: Strategies and tools for building a learning organization. London: Nicholas Bready.

Sharma, P., Chrisman, J.J. y Chua, J. H. (1997). Strategic management of the family business: Past research and future challenges. Family Business Review, 10(1), 1-35.

Simon, H. (1957). Administrative behaviour. Nueva York: MacMillan.

Sirmon, D.G. y Hitt, M.A. (2003). Managing resources: linking unique resources, management and wealth creation in family firms. Entrepreneurship: Theory and practice, 27(4), 339-358.

Stacey, R.D. (1996). Complexity and creativity in organizations. San Francisco: Berret-Koehler publishers.

Tagiuri, R. y Davis, J. (1996). Bivalent attributes of the family firm. Family Business Review, 11(2), 199-208.

Thompson, J.D. (1967). Organizations in action. Nueva York: McGraw Hill.

Tokarczyk, J., Hansen, E., Green, M. y Down, J. (2007). A resource-based view and market orientation theory examination of the role of familiness in family business success. Family Business Review, 20(1), 17-31.

Uotila, J., Maula, M., Keil, T. y Zahra, S.A. (2009). Exploration, exploitation, and financial performance: Analysis of S\&P 500 corporations. Strategic Management Journal, 30(2), 221-231.

Wang, C.L. y Ahmed, P.K. (2007). Dynamic capabilities: A review and research agenda. International Journal of Management Review, 9(1), 31-51.

Ward, J.L. (1987). Keeping the family business healthy: How to plan for continuing growth, profitability, and family leadership. San Francisco: Jossey-Bass.

Ward, J.L. (1999). Crecimiento de la empresa familiar: Los retos especiales y las mejoras prácticas. En M.A. Gallo (Ed.), La empresa familiar 6 (143-163). Barcelona: Ediciones IESE S.L.,

Youndt, M.A., Subramaniam, M. y Snell, S.A, (2004). Intellectual capital profiles: An examination of investments and returns. Journal of Management Studies, 41(2), 335-361. 